\title{
Experimental study of weak shock waves influence on the supersonic boundary layer of the flat plate model
}

\author{
Vasiliy Kocharin ${ }^{1, *}$, Aleksandr Kosinov $^{1,2}$, Yuriy Yermolayev ${ }^{1}$, and Nikolay Semionov ${ }^{1}$ \\ ${ }^{1}$ Khristianovich Institute of Theoretical and Applied Mechanics SB RAS, 630090 Novosibirsk, Russia \\ ${ }^{2}$ Novosibirsk State University, Novosibirsk, Russia
}

\begin{abstract}
The experimental study of the effect of weak shock waves on the supersonic boundary layer of the flat plate with a blunt leading edge (the radius of bluntness was $\mathrm{r}=2.5 \mathrm{~mm}$ ) with Mach number $\mathrm{M}=2.5$ and zero angle of attack was carried out. The measurements were carried out using the constant temperature anemometer. The paper presents a complex flow structure on the surface of the model. High-intensity peaks were found in the regions of the disturbed flow. Also the spectral analysis of perturbations was performed. It is found that the supersonic boundary layer on a flat plate is very sensitive to the effect of weak shock waves.
\end{abstract}

\section{Introduction}

The process of laminar-turbulent transition in supersonic boundary layers depends strongly on the level of perturbation in the free flow [1]. For example, in supersonic wind tunnels there is a high level of acoustic pulsations in the test section [2-3] causing excitation of intrinsic perturbations of the boundary layer, when development leads to an earlier laminar-turbulent transition. In the test section of the wind tunnels, the model can be affected by quasi-stationary perturbations in the form of weak shock waves.

Typical case is when a weak shock wave falls from the side wall to the leading edge of the models [4-8]. Experiments have shown that in these cases the transition in the boundary layer of models is not described itself in the framework of traditional concepts. Highintensity regions were found out in the boundary layer downstream. Up to now the mechanism of generation of such perturbations in the boundary layer is unclear.

This article is devoted to an experimental study of weak shock waves influence on the boundary layer of the flat plate model with blunting leading edge in supersonic flow.

\section{Experimental setup and data processing}

Experiments were performed in the T-325 long-duration blowdown low-noise supersonic wind tunnel, at the Khristianovich Institute of Theoretical and Applied Mechanics SB RAS, at Mach number $M=2.5$ at unit Reynolds number $\operatorname{Re}_{1}=8 \cdot 10^{6} \mathrm{~m}^{-1}$.

\footnotetext{
* Corresponding author: kocharin@itam.nsc.ru
} 


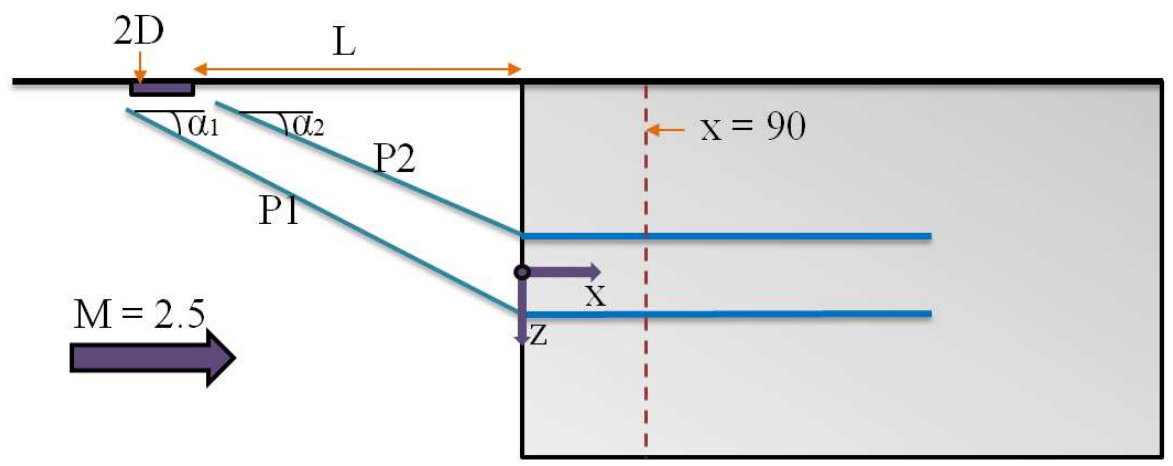

Fig. 1. Scheme of experiments on the flat plate with the blunt leading edge.

In the experiment, a stainless-steel flat plate model $440 \mathrm{~mm}$ long, $10 \mathrm{~mm}$ thick and 200 $\mathrm{mm}$ wide with a blunt leading edge with the blunting radius $\mathrm{r}=2.5 \mathrm{~mm}$ was used. In the wind tunnel the model was fixed in the central plane of the test section at approximately a zero angle of attack. The scheme of the experiment, the coordinate systems and top view are shown in Figure. 1, dimensions are given in $\mathrm{mm}$. The origin of the streamwise $\mathrm{x}$ coordinate was at the leading edge of the model and the coordinate $\mathrm{z}=0$ corresponds to the centerline along the width of the model. P1 and P2 are a pair of weak shock waves, $\mathrm{L}$ is the distance from the two-dimensional roughness to the leading edge of the plate. On the side wall of the wind tunnel test section, a two-dimensional roughness was vertically established, which was the source of the "N-wave". Use was made of sticky polyvinyl chloride tape with following dimensions: length - about $140 \mathrm{~mm}$, width $15 \mathrm{~mm}$ and thickness $0.26 \mathrm{~mm}$.

The flow disturbances were measured by a constant-temperature anemometer. The methods for determining the pulsational and mean characteristics of flow, processing methods, and setting up experiments are described in detail in [1]. Positioning accuracy of probes in $\mathrm{x}, \mathrm{z}$ directions was $0.1 \mathrm{~mm}$, and $0.01 \mathrm{~mm}$ for $\mathrm{y}$. The wire overheat ratio was installed about 0.8 , so the measured disturbances consisted from mass flow pulsations on 95\% [9]. Measurements of pulsational and mean flow characteristics were carried out by an automated measuring system described in detail in $[4,5,9]$.The value of the coordinate y for measurements by the transverse coordinate was determined from the maximum of the fluctuation inside the boundary layer when the sensor was set at the beginning of the movement along the coordinate $\mathrm{z} \approx-15 \mathrm{~mm}$, i.e. far from the incident weak shock waves, outside the area of their influence on the boundary layer.

\section{Results}

When the pair of weak shock waves hits the leading edge of the blunt plate, areas of the disturbed flow in the boundary layer above the surface of the model are detected, characterized by a change in the distribution of the mean mass flow and the root mean square pulsations of mass flow (Fig. 2). For mean mass flow and pulsations amplitude, the width of the region at a distance of $90 \mathrm{~mm}$ from the leading edge was about $35 \mathrm{~mm}$ from $\mathrm{z}$ $=-10 \mathrm{~mm}$ to $\mathrm{z}=25 \mathrm{~mm}$. In the areas of the disturbed flow, high-intensity peaks are found. The area of the disturbed flow from mass flow pulsations contains two intense peaks and one less intense peak. The left peak $(\mathrm{z} \approx-4 \mathrm{~mm})$ is generated by a weak shock wave P2. Its level reaches approximately $15 \%$. The second peak $(\mathrm{z} \approx 6 \mathrm{~mm})$ with an amplitude of approximately $10 \%$. The third, rather extended region $(15 \mathrm{~mm}<\mathrm{z}<25 \mathrm{~mm})$ is generated by a weak shock wave $\mathrm{P} 1$. The pulsation level in this region reaches $4 \%$, and the 


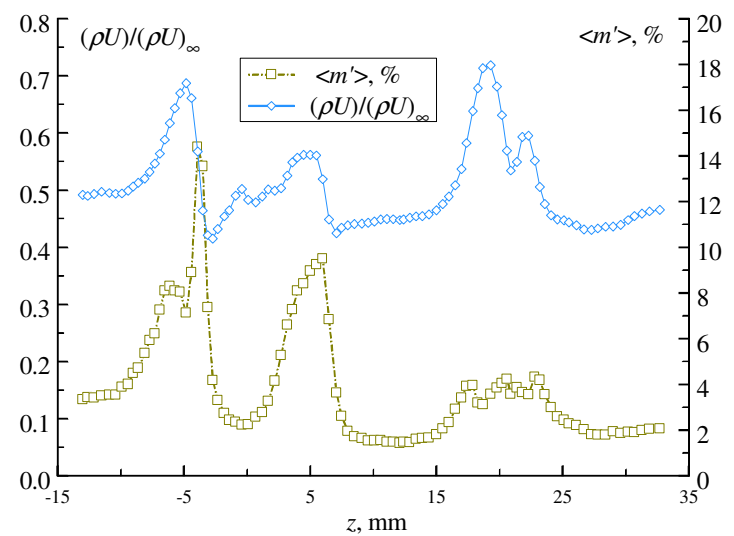

Fig. 2. Pulsation amplitude distribution $<m$ '> and value of the mean mass flow $\rho U(b)$ as a function of the transverse coordinate $\mathrm{z}$.

inhomogeneity of the mean mass flow across the transverse coordinate is more complex in comparison with the inhomogeneity of the flow generated by the weak shock wave P2. Thus, a sticker on side wall of the wind tunnel test section leads to the generation in the boundary layer of the model of high-intensity pulsations.

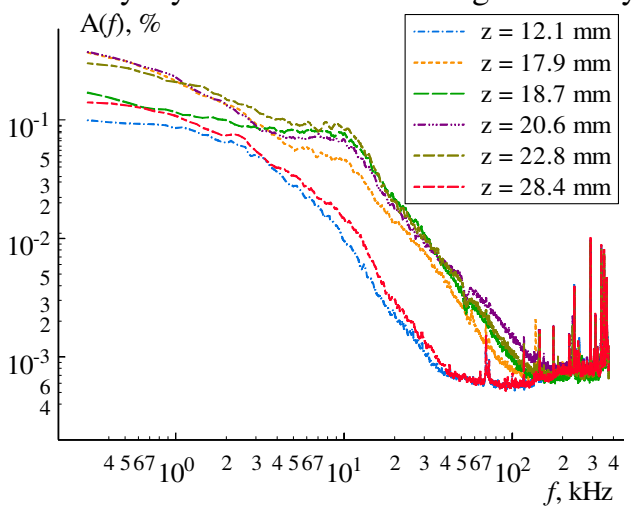

(a)

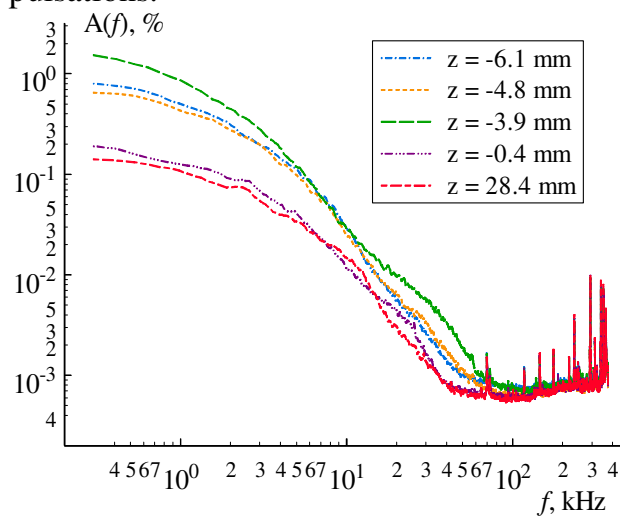

(b)

Fig. 3. The amplitude-frequency spectra of pulsations of mass flow.

Figure 3 (a) shows the amplitude-frequency spectra of mass flow pulsations in the region of influence of a weak shock wave P1 and in the unperturbed part of the boundary layer. The frequency is plotted along the abscissa axis in a logarithmic scale, and the magnitude of the mass flow pulsations is along the y-axis (also in the logarithmic scale). The spectrum at $\mathrm{z}=28.4 \mathrm{~mm}$ is measured in the region of the unperturbed boundary layer. At a value of $\mathrm{z}$ from 17.9 to 22.8 , the spectrum corresponds to the region of influence of the weak shock wave from the leading edge of $2 \mathrm{D}$ roughness $\mathrm{P} 1$. It can be noted that here amplification of the amplitude of the spectrum occurs in the frequency range from 0.4 to 80 $\mathrm{kHz}$ with respect to the amplitude of the unperturbed flow spectrum. The spectrum at $\mathrm{z}=$ $12.1 \mathrm{~mm}$ characterizes the region between the waves from the front and back edges of the two-dimensional roughness. It almost does not differ from the spectrum of the unperturbed flow.

Figure 3 (b) shows the amplitude-frequency dependences from measurements in the region of the effect of the weak shock wave $\mathrm{P} 2$ and in the unperturbed part of the boundary layer (at $\mathrm{z}=28.4$ ). The region of influence of a weak shock wave from the trailing edge of 
the $2 \mathrm{D}$ roughness $\mathrm{P} 2$ corresponds to a value of $\mathrm{z}$ from -6.3 to -0.4 . In this region, an increase in the harmonic amplitude is observed in the frequency range from 0.4 to $60 \mathrm{kHz}$ with respect to the undisturbed flow. The spectral analysis of the measured pulsations was carried out, which showed that in the regions of pulsation peaks there are nonlinear perturbations in the low-frequency part of the spectrum and in the high-frequency part.

\section{Conclusion}

It is found that the boundary layer on a flat plate with a blunt leading edge turns out to be very sensitive to the effect of weak shock waves with Mach number $M=2.5$. In the areas of the disturbed flow, high-intensity peaks are found. Also, it is found that the largest level of mass flow pulsations in the boundary layer occurred under the effect of the trailing edge of the weak shock "N-wave" and it reach the value of 15\%. The spectral analysis showed that in the regions of pulsation peaks there are nonlinear perturbations in the low-frequency part of the spectrum and in the high-frequency part.

This work is supported by the Russian Foundation for Basic (grant number 16-01-00743).

\section{References}

1. A. Vaganov, Yu. Ermolaev, G. Kolosov, A. Kosinov, A. Panina, N. Semionov and A. Yatskikh, Thermophys. Aeromech. 23(1), 43 (2016).

2. J. Laufer, J. Aerospace Sci. 28(9), 685 (1961).

3. J. M. Kendall, AIAA J. 13(3), 290 (1975).

4. A. Vaganov, Yu. Ermolaev, A. Kosinov, N. Semionov and V. Shalaev, Proceedings of MIPT, 5(3), 164 (2013).

5. A. Vaganov, Yu. Ermolaev, G. Kolosov, A. Kosinov, A. Panina, and N. Semionov, Vestnik Novosibirsk State University. Series: Physics. 9(1), 29 (2014). (in Russian).

6. A. Kosinov., M. Golubev, and A. Pavlov, Siberian journal of physics. 12(2), 20 (2017).

7. D. Khotyanovsky, A. Kudryavtsev and A. Kosinov, AIP Conference Proceeding 1893, 030051 (2017).

8. Y. Yermolaev, A. Yatskikh, A. Kosinov, and N. Semionov, AIP Conference Proceedings 1770, 020012 (2016).

9. A. Kosinov, N. Semionov and Yu. Yermolaev. Disturbances in test section of T-325 supersonic wind tunnel. Preprint ITAM SB RAS 6-99, (ITAM SB RAS, Novosibirsk, 1999). 